\title{
Modificaciones fotomorfogénicas inducidas por la calidad de la luz en plantas cultivadas
}

\author{
Fánor Casierra-Posada*, Jaime E. Peña-Olmos
}

Grupo de Investigación de Ecofisiología Vegetal, Facultad de Ciencias Agropecuarias, Universidad Pedagógica y Tecnológica de Colombia (UPTC)

\begin{abstract}
Resumen
Las longitudes de onda importantes en fotobiología son el ultravioleta, la luz visible y la infrarroja. Las longitudes de onda entre 300 y $900 \mathrm{~nm}$ son capaces de afectar el crecimiento y el desarrollo de los vegetales. Sin embargo, no solamente la calidad de luz puede influir en los procesos de crecimiento de las plantas, pues otras de sus propiedades, por ejemplo su intensidad y su duración, así como los factores climáticos, también están involucradas. El desarrollo de investigaciones con una sola franja de longitud de onda es muy útil en la identificación del espectro más adecuado para la aplicación en estudio. No obstante, la detección de las influencias de los segmentos de espectro adyacentes en ciertas aplicaciones es difícil debido a que este es continuo, sin márgenes aparentes entre los colores vecinos. El uso de coberturas de colores o filtros fotoselectivos es una técnica agrícola, que si bien no es nueva, aún puede explorarse y estudiarse para esclarecer aspectos relacionados con su uso, como los estímulos lumínicos y la respuesta de las plantas a ellos, con el objetivo de proporcionar al agricultor alternativas reales de manejo en el cultivo y, además, contribuir significativamente al desarrollo del campo de la fisiología vegetal. La investigación enfocada a la influencia de la luz sobre el crecimiento vegetal es extensa, y es necesario discutir sus hallazgos para consolidar su aporte sobre bases objetivas a la hora de hacer modificaciones en el ambiente lumínico como estrategia de manejo en los diversos cultivos.
\end{abstract}

Palabras clave: fotobiología, fotomorfogénesis, crecimiento, color.

Photomorphogenic changes induced by the quality of light in crops

\begin{abstract}
The most relevant wavelengths in photobiology are the ultraviolet, the visible light, and the infrared. Wavelengths between 300 and $900 \mathrm{~nm}$ can affect plants growth and development. Nevertheless, not only light quality can influence plant growth processes. Light properties such as its intensity and duration, as well as climatic factors, are also involved. The development of studies using a single wavelength is very useful to identify the most responsive spectrum in the target application. However, detecting the influences of adjacent spectrum segments in certain applications is difficult because the spectrum is continuous, with no apparent margins between neighboring colors. The agricultural technique of colored covering, although not new, can still be explored in order to clarify aspects concerning its use as light stimulus and the response of plants to it, and thus offer to farmers alternatives of crop management, which will also contribute significantly to the study of plant physiology. Research focused on the influence of light on plant growth is wide; however, it is necessary to discuss its findings so as to offer solid evidence if the aim is to introduce modifications in the light environment as a strategy for crop management.
\end{abstract}

Key words: Photobiology, photomorphogenesis, growth, color.

\section{Introducción}

La percepción de la luz permite a los seres vivos adaptarse a los cambios en la iluminación, e influye considerablemente en el control de la morfogénesis, la fotosíntesis y la prevención de daños causados por las radiaciones nocivas (Anders \& Essen, 2015). En su gran mayoría, las especies vegetales tienen la capacidad de responder de diferentes formas a la calidad de la luz, entendida esta como el color o la longitud de onda, a su intensidad, es decir, la densidad del flujo fotónico o irradiancia, y a la combinación de ambas.
Este aspecto es de gran relevancia en comunidades vegetales como los cultivos y los ecosistemas vegetales, debido a que las plantas absorben ciertos rangos de la radiación incidente y transmiten la radiación no absorbida a las plantas vecinas. El efecto de la calidad e intensidad lumínica se combina con el componente de sombra en la oferta ambiental y produce

\footnotetext{
*Correspondencia:

Fánor Casierra-Posada, fanor.casierra@uptc.edu.co

Recibido: 31 de agosto de 2015

Aceptado: 14 de septiembre de 2015
} 
un espectro de radiación singular en el entorno de las plantas (Nguy-Robertson, et al., 2015). Los órganos fotosintéticos de las plantas (hojas y tallos verdes) absorben los fotones en las franjas azul y roja del espectro de la radiación visible incidente, mientras que la absorción en la franja del verde y del infrarrojo es escasa, y la mayoría de estos fotones se reflejan como radiación difusa (Lazo \& Ascencio, 2010).

La eficiencia de la luz, cuya energía es almacenada en los fotosintatos luego de un complejo proceso, es una medida muy útil para determinar la productividad de las plantas cultivadas (Monteith, 1972). No obstante, la incorporación de la calidad de la luz a las mediciones de su eficiencia ha demostrado que mejora la agudeza de los modelos con los que se pretende expresar o predecir la productividad de los ecosistemas (Suyker \& Verma, 2012).

Las plantas poseen diferentes tipos de fotorreceptores que les son muy útiles para relacionarse con las variables de la oferta ambiental, y la captación de la luz les facilita el control de todos los procesos involucrados en el desarrollo y en la conservación de la homeostasis. Estos fotorreceptores son de diversos tipos y capturan la radiación en diferentes rangos del espectro. Algunos de ellos capturan la luz en el rango del UV-B, mientras que los criptocromos y las fototropinas reciben la luz en las franjas del UV-A y el azul (Štroch, et al., 2015). Las clorofilas, por su parte, absorben la radiación en las franjas del azul y el rojo, y los carotenos capturan los colores verde y amarillo (Kendrick \& Weller, 2003, Esteban, et al., 2015). Por último, los fitocromos absorben en las franjas del rojo y el infrarojo (Anders \& Essen, 2015). La acción conjunta de estos pigmentos en cuanto a sus rangos de absorción induce en las plantas las respuestas fotomorfogénicas; no obstante, entre estos grupos de fotorrecetores los fitocromos y los criptocromos son los más estudiados en relación con la fotomorfogénesis en vegetales (Yeom, et al., 2014, Zhang, et al., 2014, Ou Yang, et al., 2015).

La calidad de la radiación solar, medida en términos del color de la luz captada por las plantas, podría convertirse en una herramienta muy útil en el manejo de las plantas cultivadas y para lograr un aumento en la cantidad o en la calidad el producto cosechado (Casierra-Posada, et al., 2014a). Teniendo en cuenta que las plantas cuentan con diversas rutas para llevar a cabo su metabolismo, la captación de luz y su posterior transformación en energía química, que finalmente es distribuida a lo largo del vegetal, requiere de mecanismos complejos y fotoselectivos específicos para llevar a cabo procesos como la fotosíntesis (CasierraPosada, et al., 2011a).

El crecimiento y el desarrollo de un vegetal están influenciados, entre otros factores, por la intensidad y la calidad de la luz captada por los órganos que realizan la fotosíntesis. Cuando se generan cambios en la calidad o en la intensidad de la radiación incidente, se producen modificaciones en la planta que afectan su anatomía y fisiología, así como su crecimiento y desarrollo, fuertemente influenciados por la calidad de la luz en términos del color o la longitud de onda que llega a la superficie de las hojas (Johkan, et al., 2010, Takahashi, et al., 2012). Se ha encontrado que la calidad de la luz afecta la tasa fotosintética, la eficiencia cuántica para la asimilación del $\mathrm{CO}_{2}$ y la máxima actividad de la fosfoenolpiruvato carboxilasa. Por ejemplo, estos valores fueron significativamente inferiores en plantas de Zea mays y Miscanthus x giganteus al ser expuestas a la luz azul, en comparación con los valores obtenidos cuando las plantas se expusieron a la luz blanca, roja y verde, por lo que se concluyó que la calidad de la luz afectó la tasa de asimilación de $\mathrm{CO}_{2}$ en estas plantas, pero no la eficiencia del mecanismo de concentración del $\mathrm{CO}_{2}$ (Sun, et al., 2012).

El uso de coberturas en la propagación y producción de especies de interés agrícola es una práctica que despierta cada vez más el interés de muchos investigadores de las ciencias agrarias. Las modificaciones del ambiente lumínico en el cual la planta lleva a cabo su crecimiento y desarrollo, inducen en el vegetal varias respuestas morfogenéticas que, por un lado, contrarrestan las condiciones adversas y, por otro, promueven respuestas fisiológicas que mejoran su funcionamiento y aumentan la cantidad y la calidad del producto cosechado (Rajapakse \& Shahak, 2007, Cope \& Bugbee, 2013, Casierra-Posada, et al., 2014a)

A pesar de los muchos estudios orientados a determinar y esclarecer los efectos de la luz y su influencia sobre el crecimiento y el desarrollo de los vegetales, los resultados relativos a las diferentes respuestas de las plantas a estímulos mediados por cambios en el ambiente lumínico han sido contradictorios y, en ocasiones, poco consistentes, dado que las variables que componen la oferta ambiental son numerosas y en muchos casos, además, estas respuestas dependen de la capacidad de la planta misma para reaccionar a los cambios en su entorno. Las plantas poseen diferentes tipos de fotorreceptores, cada uno con una función definida por la longitud de onda que incide sobre la hoja (Jiao, et al., 2007, Folta \& Maruhnich, 2007, Mathews, 2010, Strasser, et al., 2010, Casierra-Posada, et al., 2014b). Del mismo modo, la eficiencia con que cada fotorreceptor es capaz de captar determinado color de luz puede medirse, indirectamente, mediante diversas metodologías. El crecimiento vegetal es uno de tales recursos, y para medirlo se cuenta con variables como el peso seco, el área foliar y los índices de crecimiento (Santos-Castellanos, et al., 2010).

En el presente manuscrito se informa a la comunidad académica acerca de los alcances del uso de la exposición de las plantas a la calidad de la luz en diferentes rangos del espectro, y se analizan las respuestas fotomorfogénicas de algunas plantas cultivadas bajo estas condiciones con base en algunos trabajos llevados a cabo en el altiplano tropical cuyos resultados se confrontan con los de investigaciones desarrolladas en diferentes latitudes. 


\section{La luz como factor de crecimiento}

La participación de la luz en el crecimiento de las plantas es compleja. Las características de la luz cambian con la longitud de onda. Hay ondas electromagnéticas perjudiciales, como los rayos cósmicos y los gamma, que pueden lesionar a los organismos vivos. Sin embargo, los seres vivos pueden utilizar la mayoría del espectro electromagnético que incide sobre la superficie de la tierra, en particular la parte visible de este espectro, que llamamos "luz". En general, se sabe que la luz visible tiene aproximadamente la misma longitud de onda que la radiación fotosintética activa (Fukuda, 2013).

La luz tiene dos funciones importantes en el crecimiento de las plantas: como fuente de energía para la fotosíntesis, pues sin su participación las plantas superiores no pueden crecer, y como un estímulo, por ejemplo, para el control del crecimiento de las plantas, o para regular el momento de la floración o la morfogénesis (Momokawa, et al., 2011, Higuchi, et al., 2012). Por lo tanto, el crecimiento de las plantas y su desarrollo a veces se alteran como respuesta a los cambios en el color de la luz irradiada (Eskins, 1992, Guo, et al., 2012, Nguy-Robertson, et al., 2015). Por otro lado, debido a la gran variación de las condiciones de iluminación natural y a su carácter sésil, las plantas se ven obligadas a aclimatarse a nivel celular y molecular ante los cambios en la oferta ambiental. Los cambios en la intensidad o en la calidad de la luz inducen alteraciones en los estados de oxidación y reducción (redox) de la cadena transportadora de electrones para la fotosíntesis, que actúa como inicio de las respuestas de aclimatación compensatorias, incluidos los ajustes funcionales y estructurales de la fotosíntesis y el metabolismo (Dong, et al., 2014, Dietzel, et al., 2015).

\section{La calidad de la luz y su influencia sobre el crecimiento vegetal}

Los filtros fotoselectivos garantizan a la planta ambientes controlados con un determinado porcentaje de opacidad (disminución de la radiación directa), el cual depende del color y de la naturaleza del material de la cobertura, y causan modificaciones con respecto a la radiación fotosintéticamente activa (PAR) disponible para la planta (CasierraPosada \& Rojas, 2009, Casierra-Posada \& Pinto-Correa, 2011, Casierra-Posada, et al., 2012a, Casierra-Posada, et al., 2012b, Casierra-Posada, et al., 2014b, CasierraPosada, et al., 2014c). En la Tabla 1 se presentan los valores de la PAR y de opacidad de diferentes tipos de coberturas utilizadas en algunos cultivos de interés agrícola.

La luz influye igualmente en el peso seco total de la planta. Las plantas de diversos cultivos de interés agrícola presentan diferencias significativas en la producción de biomasa cuando son afectadas por la calidad de la luz. Las longitudes de onda entre 400 y $550 \mathrm{~nm}$, que corresponden al rango azul-verde, han inducido una disminución en el peso seco total de la planta en algunos cultivos como el de fresa (Casierra-Posada, et al., 2012b), remolacha (CasierraPosada \& Pinto-Correa, 2011) y brócoli (Casierra-

Tabla 1. Radiación fotosintéticamente activa (PAR) y opacidad determinadas bajo filtros fotoselectivos utilizados en diferentes cultivos (Casierra-Posada \& Rojas, 2009, Casierra-Posada \& Pinto-Correa, 2011, Casierra-Posada, et al., 2012a, Casierra-Posada, et al., 2012b, Casierra-Posada, et al., 2014b, Casierra-Posada, et al., 2014c)

\begin{tabular}{|c|c|c|c|c|c|c|c|}
\hline \multicolumn{8}{|c|}{ Radiación fotosintéticamente activa - PAR $\left(\mu \mathrm{mol} \mathrm{m} \mathrm{m}^{-2} \mathrm{~s}^{-1}\right)$} \\
\hline \multicolumn{8}{|c|}{ Color de cobertura } \\
\hline & Azul & Rojo & Verde & Amarillo & Transparente & Naranja & Control \\
\hline Remolacha & 303,78 & 467,67 & - & - & 720,6 & - & 898,27 \\
\hline Acelga & 185 & 278 & 261 & 234 & 265 & - & - \\
\hline Pimentón & 486 & 578 & 452 & 376 & 622 & - & 1380 \\
\hline Fresa & 99,31 & 86,95 & 78,87 & 152,19 & 210,46 & - & 299,94 \\
\hline Brócoli & 299,45 & 425,74 & - & 356,24 & 667,29 & 423,59 & 818,12 \\
\hline Calas & 99,31 & 86,95 & 78,87 & 152,19 & 210,46 & - & 299,94 \\
\hline \multicolumn{8}{|c|}{ Opacidad (\%) } \\
\hline \multicolumn{8}{|c|}{ Color de cobertura } \\
\hline & Azul & Rojo & Verde & Amarillo & Transparente & Naranja & Control \\
\hline Remolacha & 65,72 & 48,73 & - & - & 21,39 & - & 0 \\
\hline Acelga & 62,3 & 43,5 & 46,9 & 52,4 & 46,1 & - & - \\
\hline Pimentón & 35,22 & 41,88 & 32,78 & 27,25 & 45,07 & - & 0 \\
\hline Fresa & 66,89 & 71,01 & 73,7 & 49,25 & 29,83 & - & 0 \\
\hline Brócoli & 63,4 & 48 & - & 56,5 & 18,5 & 48,3 & 0 \\
\hline Calas & 66,89 & 71,01 & 73,7 & 49,25 & 29,83 & - & 0 \\
\hline
\end{tabular}


Posada \& Rojas, 2009); sin embargo, Casierra-Posada, et al., (2012a) también encontraron que en plantas de cala, el color verde (500-550 nm) favorecía la acumulación de materia seca (Tabla 2). Xiaoying, et al., (2012) encontraron que la luz de diodo emisor de luz (light-emitting diode, LED) de color azul aumentaba el peso seco total de la planta en tomates cherry. La fotosíntesis y el crecimiento de estas plantas de tomate bajo la influencia de la luz azul, fue superior en comparación con las plantas de control. Según los autores, este resultado podría explicarse con los hallazgos de Matsuda, et al., (2008), quienes afirman que la luz azul se convierte en un estímulo para inducir respuestas de aclimatación en las plantas, encaminadas a la distribución de energía en el fotosistema II (PSII) y, a su vez, tiene que ver con la asimilación del $\mathrm{CO}_{2}$ en condiciones de alta irradiación.

En estudios llevados a cabo por Casierra-Posada, et al., (2012b), las plantas de fresa presentaron un incremento en el peso seco total de la planta cuando crecieron bajo un filtro fotoselectivo de color rojo, en comparación con los filtros de colores azul, verde y amarillo. Hultberg, et al. (2014) encontraron que al exponer la microalga Chlorella vulgaris a luces monocromáticas de colores amarillo, rojo y blanco, la biomasa total acumulada se incrementó en comparación con las microalgas expuestas a luces de colores azul, verde y púrpura. Del mismo modo, Rodríguez \& Lazo, (2012) encontraron que 35 días después de la siembra, las plantas de coquito (Cyperus rotundus L.) presentaron un aumento en la masa seca total al crecer expuestas a un filtro de papel celofán de color rojo en comparación con los filtros de colores verde y blanco.

Por otro lado, Casierra-Posada, et al., (2011) encontraron que la relación $F_{\mathrm{v}} / F_{\mathrm{m}}$, o eficiencia cuántica máxima del PSII, disminuyó significativamente en las hojas de las plantas que crecieron bajo filtros fotoselectivos de colores amarillo y transparente comparadas con las plantas expuestas a coberturas de colores azul, verde y roja. Según los autores, este resultado se debió al aumento de la fluorescencia inicial $\left(\mathrm{F}_{0}\right)$ causado por una elevada reducción del primer receptor de electrones (el pool de quinonas $\mathrm{A}_{\mathrm{A}} \mathrm{Q}_{\mathrm{A}}$ ), lo que influyó directamente en una variación en la tasa de transporte de electrones y, por ende, modificó el rendimiento fotosintético del vegetal, mientras que los altos valores de esta misma variable en las coberturas de colores azul, verde y rojo se relacionaron con la eficiencia de los mecanismos fotoprotectores de la planta que se encargan de modular la probabilidad de los daños ocasionados por la luz. Por otro lado, Slauenwhite \& Qaderi, (2013) encontraron que en Brassica napus un valor bajo de la relación entre el rojo y el rojo lejano reducía no solo el valor del coeficiente $F_{v}{ }_{v}$ $F_{m}$ sino también los contenidos de las clorofilas a y b y de los carotenos.

Estos hallazgos demuestran que la exposición de las pantas a diferentes ambientes lumínicos mediada ya sea por filtros fotoselectivos o por fuentes de luz adicional, modifica el rendimiento fotoquímico de los vegetales y su eficiencia fotosintética, lo que incide directamente en la síntesis de fotoasimilados y, por ende, en el peso seco total de la planta.

Cuando el ambiente lumínico sufre modificaciones las plantas utilizan diversas estrategias para contrarrestar algunas condiciones que pueden considerarse adversas dependiendo del estado fisiológico del vegetal. En el caso de las coberturas de colores rojo, azul y verde, la expansión del área foliar podría estar relacionada con el porcentaje de opacidad en cada uno de los filtros. En los estudios de Casierra-Posada, et al., (2012b) en la fresa, la mayor área foliar la presentaron las plantas que crecieron bajo la cobertura de color rojo (Tabla 3), con una opacidad cercana a $71 \%$; sin embargo, en ese mismo experimento las plantas de fresa que crecieron bajo la cobertura verde, con un porcentaje de opacidad de $73,7 \%$, presentaron valores inferiores de área foliar. Para el caso de las plantas de remolacha (Casierra-Posada \& Pinto-Correa, 2011) y brócoli (Casierra-Posada \& Rojas, 2009), los investigadores encontraron que los porcentajes de opacidad de los filtros fotoselectivos de color azul indujeron mayores valores de área foliar en comparación con los filtros de color rojo; sin embargo, la expansión del área foliar de las plantas que crecieron bajo la cobertura roja fue la más alta en comparación con las demás coberturas de colores y el control sin cobertura (Tabla 3).

Tabla 2. Peso seco total de la planta en cinco diferentes cultivos de importancia agrícola expuestos a diferente calidad de luz (CasierraPosada \& Rojas, 2009), Casierra-Posada \& Pinto-Correa, 2011, Casierra-Posada, et al., 2012a, Casierra-Posada, et al., 2012b, Casierra-Posada, et al., 2014b)

\begin{tabular}{lccccccc}
\hline \multicolumn{7}{c}{ Peso seco total (g) } \\
\hline & Czul & Rojo & Verde & Amarillo & Transparente & Control & Naranja \\
\hline Acelga & 2,44 & 2,76 & 2,87 & 4,80 & 2,38 & - & - \\
Remolacha & 18,12 & 30,99 & - & - & 27,42 & - & - \\
Fresa & 21,96 & 36,44 & 6,33 & 34,69 & 37,60 & 40,59 & 91,74 \\
Brócoli & 45,57 & 106,62 & - & 70,01 & 80,75 & 88,97 \\
Cala & 26,48 & 36,07 & 41,31 & 32,37 & 40,81 & 37,71 & - \\
\hline
\end{tabular}


Tabla 3. Área foliar determinada en cinco cultivos de importancia agronómica expuestos a diferente calidad de luz (Casierra-Posada $\&$ Rojas, 2009, Casierra-Posada \& Pinto-Correa, 2011, Casierra-Posada, et al., 2012a, Casierra-Posada, et al., 2012b, Casierra-Posada, et al., 2014b).

\begin{tabular}{|c|c|c|c|c|c|c|c|}
\hline \multicolumn{8}{|c|}{ Área foliar $\left(\mathrm{cm}^{2}\right)$} \\
\hline \multicolumn{8}{|c|}{ Color de cobertura } \\
\hline & Azul & Rojo & Verde & Amarillo & Transparente & Control & Naranja \\
\hline Acelga & 319,84 & 356,70 & 357,88 & 817,87 & 177,34 & & \\
\hline Remolacha & 1032,80 & 1489,93 & & & 1268,51 & 1218,40 & \\
\hline Fresa & 768,74 & 928,37 & 348,23 & 776,89 & 487,26 & 527,14 & \\
\hline Brócoli & 232,56 & 580,17 & & 341,03 & 446,8 & 498,79 & 486,89 \\
\hline Calas & 350,50 & 490,21 & 727,58 & 381,70 & 505,60 & 640,21 & \\
\hline
\end{tabular}

El mejor desarrollo foliar en las plantas evaluadas probablemente se debió al efecto Emerson, ya que bajo los filtros fotoselectivos rojos, las longitudes de onda están en el rango de los 670 a los $700 \mathrm{~nm}$, lo cual supondría un aumento en la tasa de fotosíntesis bajo dicha cobertura. Por otro lado, la luz roja es un componente del espectro visible y este solo hecho es suficiente para que la planta crezca y realice su proceso fotosintético (Lu, et al., 2012, Olle \& Virsile, 2013).

En Cyperus rotundus el área foliar aumentó a los 21 y 28 días después de la siembra en las plantas que crecieron bajo una película de papel celofán rojo, en comparación con las plantas que crecieron bajo filtros de celofán de colores blanco y verde (Rodríguez \& Lazo, 2012). Stutte, et al.,(2009) encontraron que al combinar luces LED de longitud de onda en el rango del rojo y rojo lejano, la biomasa vegetal y la longitud de las hojas de lechuga se incrementaron, pero disminuyó la concentración de clorofila, antocianinas y carotenoides. Del mismo modo, el incremento en el crecimiento de plantas de lechuga bajo luces LED del espectro rojo lejano se relacionó estrechamente con un aumento en el área foliar y, por ende, una mayor intercepción de luz solar (Kubota, et al., 2012).

Ayala-Tafoya, et al., (2015) encontraron que el área foliar en plantas de pepino aumentó considerablemente cuando crecían bajo una malla de polisombra roja, efecto que probablemente se debió a los incrementos en los flujos de radiación total, de radiación fotosintéticamente activa y de luz roja determinados en el mismo estudio. El incremento en el área foliar suele estar relacionado con incrementos en la producción, ya que a mayor área foliar, mayor es la capacidad del vegetal para captar la luz, lo que permite obtener una mayor fuente de fotoasimilados destinados al abastecimiento de los órganos vertedero, como los frutos.

La luz puede utilizarse para mejorar la calidad nutricional de los vegetales e incrementar la producción en plantas. El uso de luces LED afecta de manera diferenciada el metabolismo de las plantas y puede originar cambios en la acumulación de azúcares en las hojas, lo cual sería un parámetro deseable en términos de calidad nutricional de los alimentos. Lin, et al., (2013) reportan que en plantas de lechuga influenciadas por luces LED de colores rojo, azul, blanco, el contenido de sólidos solubles totales aumentó en comparación con las plantas expuestas a luz fluorescente y de color rojo.

Al exponer las plantas a ambientes previamente modificados por coberturas de colores, su funcionamiento se ve influenciado de distintas formas, lo que afecta igualmente su crecimiento y desarrollo. Cuando el crecimiento vegetativo incrementa con determinado color de cobertura, generalmente aumentan también la producción y la calidad del producto cultivado, debido, ante todo, al efecto positivo que genera la calidad de la luz disponible y la eficiencia fotosintética del vegetal bajo esas condiciones en particular. Cuando se presenta un incremento en el área foliar en un vegetal, se asume que dicho comportamiento fisiológico es una estrategia de la planta para aumentar la superficie fotosintética y ser más eficaz en la captación de energía lumínica, lo que finalmente redundará en un incremento en la producción y la calidad del producto (Casierra-Posada, et al., 2011). Casierra-Posada, et al., (2011b) encontraron que bajo las coberturas de colores rojo y amarillo, los valores de acidez titulable en frutos de fresa disminuyeron en comparación con las plantas de control sin cobertura, mientras que el índice de madurez aumentó. En ese mismo estudio, los autores encontraron que los frutos que crecieron bajo la cobertura roja fueron más largos y más anchos que los de las plantas de control que crecieron sin cobertura.

La tasa de crecimiento relativo (TCR) también se vio notablemente influenciada por los filtros fotoselectivos $\mathrm{y}$, además, la longitud de onda afectó el crecimiento de los vegetales dependiendo del cultivo evaluado. En la acelga la cobertura amarilla incrementó el valor de esta variable en comparación con los demás filtros; en las calas la cobertura verde presentó los valores más altos, y en fresa la luz roja mostró diferencias con respecto a las demás coberturas (Tabla 4). En cuanto a la tasa de crecimiento absoluto (TCA), las plantas de fresa presentaron los valores más altos en el control, seguidas por las plantas desarrolladas bajo las coberturas de colores transparente y rojo. En la acelga y las calas la tendencia fue la misma que en la de los valores de TCR registrados, siendo las plantas de acelga expuestas a 
Tabla 4. Índices de crecimiento determinados en tres cultivos de interés agronómico expuestos a diferente calidad de luz (CasierraPosada, et al., 2012a, Casierra-Posada, et al., 2012b, Casierra-Posada, et al., 2014b)

\begin{tabular}{|c|c|c|c|c|c|c|}
\hline \multicolumn{7}{|c|}{ Tasa de crecimiento relativo $\left(\mathrm{g} \mathrm{g}^{-1} \mathrm{~d}^{-1}\right)$} \\
\hline \multicolumn{7}{|c|}{ Color de cobertura } \\
\hline & Azul & Rojo & Verde & Amarillo & Transparente & Control \\
\hline Fresa & 0,00640 & 0,00787 & 0,00400 & 0,00723 & 0,00777 & 0,00781 \\
\hline Acelga & 0,02260 & 0,02350 & 0,02390 & 0,02740 & 0,02220 & \\
\hline Calas & 0,00260 & 0,00330 & 0,00360 & 0,00310 & 0,00350 & 0,00340 \\
\hline \multicolumn{7}{|c|}{ Tasa de asimilación neta $\left(\mathrm{g} \mathrm{m}^{-2} \mathrm{~d}^{-1}\right)$} \\
\hline \multicolumn{7}{|c|}{ Color de cobertura } \\
\hline & Azul & Rojo & Verde & Amarillo & Transparente & Control \\
\hline Fresa & 1,26536 & 1,80774 & 0,75433 & 2,00601 & 3,49125 & 3,56716 \\
\hline Acelga & 0,00040 & 0,00043 & 0,00046 & 0,00039 & 0,00058 & \\
\hline Calas & 1,65850 & 2,04970 & 1,84680 & 2,09060 & 2,26600 & 1,84090 \\
\hline \multicolumn{7}{|c|}{ Tasa de crecimiento absoluto $\left(\mathrm{g} \mathrm{d}^{-1}\right)$} \\
\hline \multicolumn{7}{|c|}{ Color de cobertura } \\
\hline & Azul & Rojo & Verde & Amarillo & Transparente & Control \\
\hline Fresa & 0,09698 & 0,16460 & 0,02523 & 0,15551 & 0,16963 & 0,18320 \\
\hline Acelga & 0,03900 & 0,04430 & 0,04620 & 0,07820 & 0,03800 & \\
\hline Calas & 0,08610 & 0,13180 & 0,15480 & 0,11550 & 0,15270 & 0,14170 \\
\hline
\end{tabular}

la cobertura amarilla y las plantas de calas expuestas a la cobertura verde las de mayor TCA. La tasa de asimilación neta (TAN) es una medida indirecta de la eficiencia fotosintética en la planta. En ensayos con plantas de fresa, acelga y calas, la cobertura transparente mostró muy buenos valores, los más altos para el caso de la acelga y las calas, y el segundo más alto para el cultivo de fresa después del control sin cobertura.

Según Lin, et al., (2013) y Li, et al., (2013) la luz blanca combinada con luz roja y luz azul puede ser una buena estrategia de manejo para muchos cultivos en ambientes controlados, como en el caso de cultivos bajo invernadero o en cultivos in vitro. Del mismo modo, Lazo \& Ascencio (2010), Casierra-Posada, et al., (2012a) y Kurepin, et al., (2015) han afirmado que las plantas absorben fotones en los rangos del azul y el rojo del espectro, que la absorción de fotones en el rango del verde y el rojo lejano es muy débil, y que la mayoría de esos fotones son reflejados por las plantas en forma de radiación difusa.

En los estudios enfocados a evaluar la influencia de la luz sobre el crecimiento y el desarrollo de los vegetales, es común encontrar contradicciones entre los autores, lo que explicaría que las plantas de fresa, acelga, remolacha, calas y brócoli presentaran diferentes respuestas de acuerdo a cada longitud de onda del espectro lumínico en el cual se desarrolló el vegetal. Al respecto Liu, et al., (2011) y Casierra-Posada, et al., (2014b) coinciden en afirmar que muchos de los estudios relacionados con la calidad de la luz y el espectro lumínico son inconsistentes en cuanto a las intensidades de la luz, las cuales no son uniformes, muy probablemente porque los investigadores son incapaces de modular y cuantificar los parámetros de la energía espectral, lo que dificulta la comparación de los resultados.

\section{Conclusiones}

Los vegetales reaccionan de diferente manera a la radiación incidente. De igual forma, la calidad de la luz y la irradiancia tienen notables efectos morfogénicos sobre ellos. Estos dos factores inducen modificaciones en su morfología y fisiología que determinan su adaptación a los componentes de la oferta ambiental. La calidad de la luz altera, además, la cantidad y la calidad del producto de la cosecha. Es así como los metabolitos producidos bajo estas condiciones se pueden ver alterados en cuanto a su contenido y actividad. De igual forma, el crecimiento se ve fuertemente influenciado por la calidad de la luz, lo cual puede ser una consecuencia de las modificaciones en el área foliar o en la producción de biomasa. La morfología del vegetal sufre cambios en respuesta a la calidad de la luz, lo cual se refleja en la altura de las plantas o en el grosor de sus órganos. A pesar de que la influencia de la calidad de la luz es evidente, no existe un modelo de respuesta en la mayoría de las plantas cuando se las expone a rangos específicos de longitud de onda, dado que al comparar los hallazgos de investigaciones llevadas a cabo bajo condiciones similares, se observan grandes contrastes cuando diferentes especies se exponen a la misma franja de longitud de onda. Este resultado sugiere que las 
plantas reaccionan a la calidad de la luz de acuerdo con las señales provenientes de los pigmentos fotorreceptores. No obstante las diferencias de los resultados en las distintas especies, la exposición de las plantas a la luz enriquecida con una franja determinada del espectro continúa siendo una herramienta muy útil que debe aprovecharse para obtener un mejor producto cosechado.

\section{Agradecimientos}

Los estudios realizados por los autores, que fueron la base para la redacción del presente manuscrito, se desarrollaron con el apoyo de la Dirección de Investigaciones de la Universidad Pedagógica y Tecnológica de Colombia en el marco del plan de trabajo del Grupo de Investigación de Ecofisiología Vegetal adscrito al programa de Ingeniería Agronómica de la Facultad de Ciencias Agropecuarias.

\section{Conflicto de interés}

El autor declara que no tiene conflicto de interés

\section{Bibliografía}

Anders, K., Essen, L.-O. (2015). The family of phytochromelike photoreceptors: Diverse, complex and multi-colored, but very useful. Current Opinion in Structural Biology. 35: $7-16$.

Ayala-Tafoya, F., Yáñez-Juárez, M.G., Partida-Ruvalcaba, L., Ruiz-Espinosa, F.H., Campos-García, H., VásquezMartínez, O., Velázquez-Alcaraz, T. de J., Díaz-Valdés, T. (2015). Producción de pepino en ambientes diferenciados por mallas de sombreo fotoselectivo. Información Técnica Económica Agraria. 111 (1): 3-17.

Casierra-Posada, F. \& Rojas, J.F. (2009). Efecto de la exposición del semillero a coberturas de colores sobre el desarrollo y productividad del brócoli (Brassica oleracea var. italica). Agronomía Colombiana. 27 (1): 49-55.

Casierra-Posada, F. \& Pinto-Correa, J.R. (2011). Crecimiento de plantas de remolacha (Beta vulgaris L. var. Crosby Egipcia) bajo coberturas de color. Revista Facultad Nacional de Agronomía Medellín. 64 (2): 6081-6091.

Casierra-Posada, F., Peña-Olmos, J.E., Ulrichs, C. (2011a). Crecimiento y eficiencia fotoquímica del fotosistema II en plantas de fresa (Fragaria sp.) afectadas por la calidad de la luz: implicaciones agronómicas. Revista U.D.C.A Actualidad \& Divulgación Científica. 14 (2): 43-53.

Casierra-Posada, F., Peña-Olmos, J.E., Vargas-Martínez, A.F. (2011b). Propiedades fisicoquímicas de fresas (Fragaria sp) cultivadas bajo filtros fotoselectivos. Revista Facultad Nacional de Agronomía Medellín. 64 (2): 6221-6228.

Casierra-Posada, F., Nieto, P.J., Ulrichs, C. (2012a). Crecimiento, producción y calidad de flores en calas (Zantedeschia aethiopica (L.) K. Spreng) expuestas a diferente calidad de luz. Revista U.D.C.A Actualidad \& Divulgación Científica. 15 (1): 97-105.

Casierra-Posada, F., Peña-Olmos, J.E., Ulrichs, C. (2012b). Basic growth analysis in strawberry plants (Fragaria sp.) exposed to different radiation environments. Agronomía Colombiana. 30 (1): 25-33.

Casierra-Posada, F., Peña-Olmos, J.E., Zapata-Casierra, E. 2014a. Pigment content in strawberry leaves (Fragaria sp.) exposed to different light quality. Revista U.D.C.A Actualidad \& Divulgación Científica. 17 (1): 87-94.

Casierra-Posada, F., Zapata-Casierra, E., Chaparro-Chaparro, D.A. (2014b). Growth analysis in chard plants (Beta vulgaris L. Cicla, cv. Pencas Blancas) exposed to different light quality. Agronomía Colombiana. 32 (2): 205-212.

Casierra-Posada, F., Matallana-Díaz, Y.A., \& Zapata-Casierra, E. (2014c). Growth of bell pepper plants (Capsicum annuum) affected by coloured covers. Gesunde Pflanzen. 66 (4): 149-155.

Cope, K.R., \& Bugbee, B. (2013). Spectral effects of three types of white light-emitting diodes on plant growth and development: Absolute versus relative amounts of blue light. HortScience. 48 (4): 504-509.

Dietzel, L., Gläßer, C., Liebers, M., Hiekel, S., Courtois, F., Czarnecki, O., Schlicke, H., Zubo, Y., Börner, T., Mayer, K., Grimm, B., \& Pfannschmidt, T. (2015). Identification of early nuclear target genes of plastidial redox signals that trigger the long-term response of Arabidopsis to light quality shifts. Molecular Plant. 8 (8):1237-1252.

Dong. C., Fu, Y., Liu, G., Liu, H. (2014). Growth, photosynthetic characteristics, antioxidant capacity and biomass yield and quality of wheat (Triticum aestivum L.) exposed to LED light sources with different spectra combinations. Journal of Agronomy and Crop Science. 200 (3): 219230.

Eskins, K. (1992). Light quality effects on Arabidopsis development. Red, blue and far-red regulation of flowering and morphology. Physiologia Plantarum. 86: 439-444.

Esteban, R., Morán, J.F., Becerril, J.M., García-Plazaola, J.I. (2015). Versatility of carotenoids: An integrated view on diversity, evolution, functional roles and environmental interactions. Environmental and Experimental Botany. 119: $63-75$.

Folta, K.M., \& Maruhnich, S.A. (2007). Green light: A signal to slow down or stop. Journal of Experimental Botany. 58: 3099-3111.

Fukuda, N. (2013). Advanced light control technologies in protected horticulture: A review of morphological and physiological responses in plants to light quality and its application. Journal of Developments in Sustainable Agriculture. 8: 32-40.

Guo, B. A., Mu, Y.C., Wang, F., Dong, S. L. (2012). Effect of periodic light color change on the molting frequency and growth of Litopenaeus vannamei. Aquaculture. 362: 67-71.

Higuchi, Y., Sumitomo, K., Oda, A., Shimizu, H., \& Hisamatsu, T. (2012). Day light quality affects the night-break response in the short-day plant chrysanthemum, suggesting differential phytochrome-mediated regulation of flowering. Journal of Plant Physiology. 169: 1789-1796. 
Hultberg, M., Larsson, H., Bergstrand, K.J., Carlsson, A.S. (2014). Impact of light quality on biomass production and fatty acid content in the microalga Chlorella vulgaris. 159: 465-467.

Jiao, Y., Lau, O.S., Deng, X.W. (2007). Light-regulated transcriptional networks in higher plants. Nature Reviews Genetics. 8: 217-230.

Johkan, M., Shoji, K., Goto, F., Hashida, S., Yoshihara, T. (2010). Blue light-emitting diode light irradiation of seedlings improves seedling quality and growth after transplanting in red leaf lettuce. HortScience. 45: 1809-1814.

Kendrick, R.E. \& Weller J.L. (2003). Regulators of growth / Phytochromes and other photoreceptors. Encyclopedia of Applied Plant Sciences. P. 1063-1069.

Kubota, C., Chia, P., Yang, Z., Li, Q. (2012). Application of far-red light emitting diodes in plant production under controlled environments. Acta Horticulturae. 952: 59-66.

Kurepin, L.V., Pharis, R.P., Emery, R.J.N., Reid, D.M., Chinnappa, C.C. (2015). Phenotypic plasticity of sun and shade ecotypes of Stellaria longipes in response to light quality signaling, gibberellins and auxin. Plant Physiology and Biochemistry. 94: 174-180.

Lazo, J.V., \& Ascencio, J. (2010). Efecto de diferentes calidades de luz sobre el crecimiento de Cyperus rotundus. Bioagro. 22 (2): 153-158.

Li, H., Tang. C., Zu, Z. (2013). The effects of different light qualities on rapeseed (Brassica napus L.) plantlet growth and morphogenesis in vitro. Scientia Horticulturae. 150: 117-124.

Lin, K.-H., Huang, M.Y., Huang, W.D., Hsu, M.H., Yang, Z.W., Yang, C.M. (2013). The effects of red, blue, and white light-emitting diodes on the growth, development, and edible quality of hydroponically grown lettuce (Lactuca sativa L. var. Capitata). Scientia Horticulturae. 150: 86-91.

Lu, N., Maruo, T., Johkan, M., Hohjo, M., Tsukagoshi, S., Ito, Y., Ichimura, T., Shinohara, Y. (2012). Effects of supplemental lighting with light-emitting diodes (LEDs) on tomato yield and quality of single-truss tomato plants grown at high planting density. Environmental Control in Biology. 50 (1): 63-74.

Liu, M., Xu, Z., Yang, Y. (2011). Effects of different spectral lights on Oncidium PLBs induction, proliferation, and plant regeneration. Plant Cell, Tissue and Organ Culture. 106: $1-10$.

Mathews, S. (2010). Evolutionary studies illuminate the structuralfunctional model of plant phytochromes. The Plant Cell. 22: 4-16.

Matsuda, R., Ohashi-Kaneko, K., Fujiwara, K., Kurata, K. (2008). Effects of blue light deficiency on acclimation of light energy partitioning in PSII and $\mathrm{CO}_{2}$ assimilation capacity to high irradiance in spinach leaves. Plant and Cell Physiology. 49: 664-670.

Monteith, J.L. (1972). Solar radiation and productivity in tropical ecosystems. Journal of Applied Ecology. 9: 747-766.
Momokawa, N., Kadono, Y., Kudoh, H. (2011). Effects of light quality on leaf morphogenesis of a heterophyllous amphibious plant, Rotala hippuris. Annals of Botany. 108: 1299-1306.

Nguy-Robertson, A., Suyker, A., Xiangming X. (2015). Modeling gross primary production of maize and soybean croplands using light quality, temperature, water stress, and phenology. Agricultural and Forest Meteorology. 213: $160-172$

Olle, M., \& Virsile, A. (2013). The effect of light-emitting diode lighting on greenhouse plant growth and quality. Agricultural and Food Science. 22: 223-234.

OuYang, F., Mao, J.-F., Wang, J., Zhang, S., Li, Y. (2015). Transcriptome analysis reveals that red and blue light regulate growth and phytohormone metabolism in Norway spruce [Picea abies (L.) Karst.]. PLoS ONE. 10 (8): 1-19.

Rajapakse, N.C., \& Shahak, Y. (2007). Light-quality manipulation by horticulture industry. En: Whitelam, G.C.; Halliday, K.J. (editors). Light and plant development. Blackwell Publ. Oxford, UK. p. 290-312.

Rodríguez, N. \& Lazo, J.V. (2012). Efecto de la calidad de luz sobre el crecimiento del corocillo (Cyperus rotundus L.). Revista Científica UDO Agrícola. 12 (1): 74-82.

Santos-Castellanos, M., Segura-Abril, M., Nustez-López, C.E. (2010). Análisis de crecimiento y relación fuente-demanda de cuatro variedades de papa (Solanum tuberosum L.) en el municipio de Zipaquirá (Cundinamarca, Colombia). Revista Facultad Nacional de Agronomía Medellín. 63 (1): 5253-5266.

Slauenwhite, K.L.I. \& Qaderi, M.M. (2013). Single and interactive effects of temperature and light quality on four canola cultivars. Journal of Agronomy and Crop Science. 199: $286-298$.

Štroch, M., Materová, Z., Vrábl, D., Karlický, V., Šigut, L., Nezval, J., Špunda, V. (2015). Protective effect of UV-A radiation during acclimation of the photosynthetic apparatus to UV-B treatment. Plant Physiology and Biochemistry. 96: 90-96.

Stutte, G.W., Edney, S., Skerritt, T. (2009). Photoregulation of bioprotectant content of red leaf lettuce with light-emitting diodes. HortScience. 44: 79-82.

Sun, W., Ubierna, N., Ma, J.Y., Cousins, A.B. (2012). The influence of light quality on $\mathrm{C} 4$ photosynthesis under steady-state conditions in Zea mays and Miscanthus x giganteus: Changes in rates of photosynthesis but not the efficiency of the $\mathrm{CO}_{2}$ concentrating mechanism. Plant, Cell and Environment. 35: 982-993.

Suyker, A.E., \& Verma, S.B. (2012). Gross primary production and ecosystem respiration of irrigated and rainfed maizesoybean cropping systems over 8 years. Agricultural and Forest Meteorology. 165: 12-24.

Strasser, B., Sánchez-Lamas, M., Yanovsky, M.J., Casal, J.J., Cerdán, P.D. (2010). Arabidopsis thaliana life without phytochromes. Proceedings of the National Academy of Sciences. 107: 4776-4781. 
Takahashi, H., Yamada, H., Yoshida, C., Imamura, T. (2012). Modification of light quality improves the growth and medicinal quality of clonal plantlets derived from the herbal plant Gentiana. Plant Biotechnology. 29: 315-318.

Xiaoying, L., Shirong, G., Taotao, C., Zhigang, X., Tezuka, T. (2012). Regulation of the growth and photosynthesis of cherry tomato seedlings by different light irradiations of light emitting diodes (LED). African Journal of Biotechnology. 11 (22): 6169-6177.
Yeom, M., Kim, H., Lim, J., Shin, A.Y., Hong, S., Kim, J., Nam, H,G. (2014). How do phytochromes transmit the light quality information to the circadian clock in Arabidopsis? Molecular Plant. 7 (11): 1701-1704.

Zhang, Z., Ji, R., Li, H., Zhao, T., Liu, J., Lin, C., Liu, B. (2014). CONSTANS-LIKE 7 (COL7) is involved in phytochrome $\mathrm{B}$ (PhyB)-mediated light-quality regulation of auxin homeostasis. Molecular Plant. 7 (9): 1429-1440.

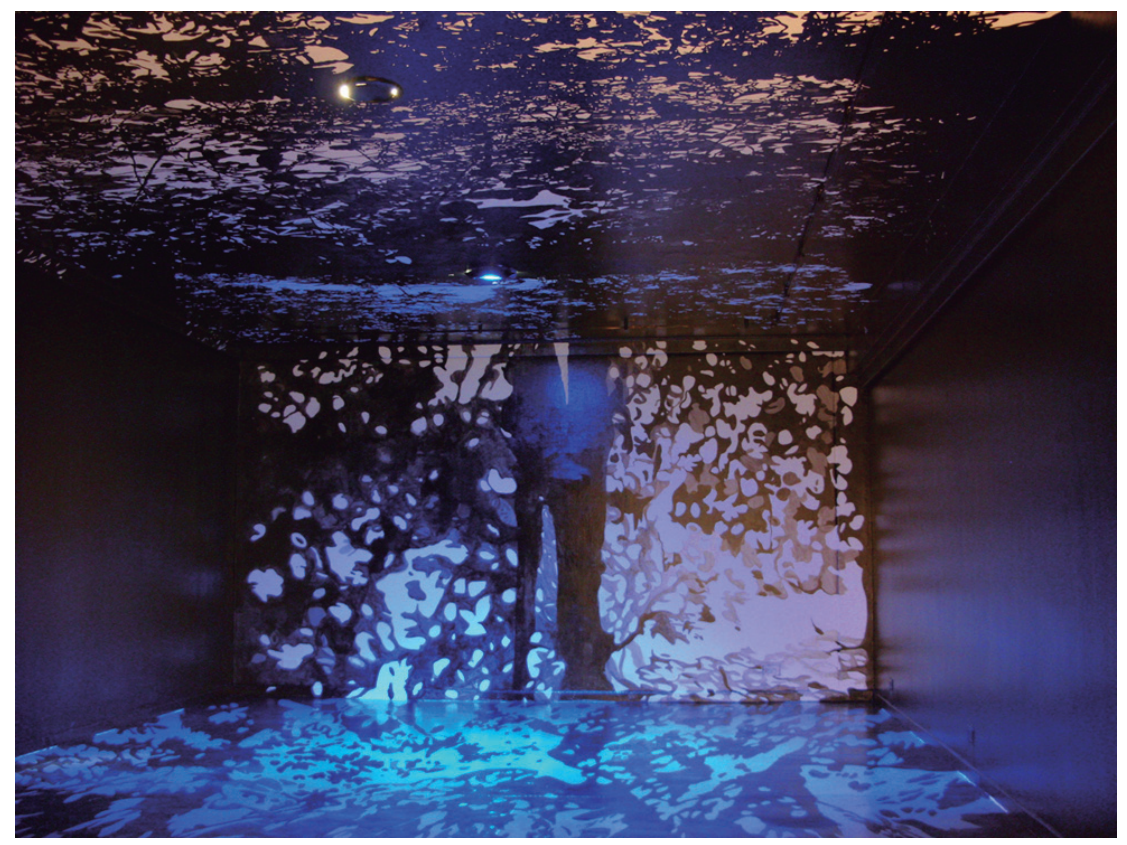

Silencio:

una hoja se hunde en el agua clara.

Issa Kobayashi (1763 - 1827) 